\title{
L'élaboration des plans de sauvegarde de l'emploi : l'intervention d'acteurs tiers ? le rôle des DDTEFP ${ }^{1}$ Philippe Chapellier, Claude Fabre, CREGOR
}

Les restructurations d'entreprises sont continues et multisectorielles. Leurs impacts sociaux sont lourds. Les plus visibles sont les licenciements collectifs, parfois accompagnés de Plans de Sauvegarde de l'Emploi (PSE). Les transitions induites comportent des risques élevés pour les individus (Gazier et Schmid, 2002). En période de crise, le retour à l'emploi des personnes licenciées est difficile. L'accompagnement social des restructurations, dont le rôle est de sécuriser les transitions en aidant les individus à élaborer un nouveau projet professionnel, est donc une priorité. Nous nous intéressons ici aux PSE, mis en œuvre en cas de " grands» licenciements pour motif économique. S'ils ne bénéficient qu'à une minorité des personnes perdant leur emploi en France, les dispositifs, moyens et procédures propres aux PSE, peuvent favoriser un accompagnement renforcé et personnalisé des restructurations pour en réduire les impacts humains et éclairer la réflexion sur la sécurisation des transitions professionnelles. Dans la continuité des travaux menés en France notamment par Beaujolin-Bellet et Bruggeman et du programme européen MIRE (Monitoring Innovative Restructuring in Europe), cet article se focalise sur la régulation sociale des PSE favorisant des restructurations « responsables » et «innovantes ». La littérature pose l'enjeu d'une régulation collective, justifiée par la place à donner aux parties affectées, par la légitimation du projet et des mesures d'accompagnement, par l'instauration d'une gouvernance équilibrée, et par les compétences nécessaires à l'élaboration et la mise en œuvre du PSE. Aux côtés de la direction et des représentants du personnel (RP), la procédure obligatoire voit intervenir des acteurs-tiers. Parmi eux, la DDTEFP, autorité administrative compétente, peut jouer un rôle-clé, qui reste pourtant méconnu. Sans négliger le contexte et les jeux d'acteurs où elle s'inscrit, nous nous penchons sur son action. La question posée est double : dans quelle mesure et sous quelles conditions son intervention contribue-t-elle à la régulation et au contenu des PSE ? Pour y répondre, trois PSE sont analysés. Après avoir réfléchi aux apports des acteurs-tiers dans la régulation sociale des PSE et exposé la démarche adoptée (partie 1), nous examinons le rôle de la DDTEFP dans les cas traités (partie 2).

\section{Des acteurs-tiers pour une meilleure régulation des PSE ?}

La littérature montre l'importance des jeux d'acteurs dans le pilotage des restructurations. Dans ce contexte, quel est l'apport des acteurs-tiers intervenant lors de l'élaboration des PSE, en particulier la DDTEFP compétente ? Partant d'un état de l'art, nous exposons et justifions la problématique et la méthodologie retenues.

\subsection{L'enjeu d'un pilotage collectif des restructurations et des PSE}

Les restructurations affectent différentes parties prenantes, au premier rang desquelles les propriétaires, dirigeants et salariés des entreprises concernées. A ce titre, le Code du Travail prévoit en cas de PSE une procédure d'information-consultation, suivie par la DDTEFP, qui met face à face la direction de l'entreprise (ou du site) et le comité d'entreprise (ou d'établissement). Cette procédure est souvent stérile ; la littérature souligne l'enjeu d'une régulation collective, mais le contexte la compromet.

\footnotetext{
${ }^{1}$ Direction Départementale du Travail, de l'Emploi et de la Formation Professionnelle
} 
La notion de régulation a plusieurs sens. Elle recouvre les mécanismes visant à mesurer, contrôler, corriger le fonctionnement d'un système, le partage du pouvoir ou les interactions entre acteurs d'un système social. Nous retenons cette dernière définition, en nous appuyant sur Reynaud (1997). L'auteur aborde la régulation sociale comme l'ensemble des échanges, formels et informels, par lesquels les acteurs élaborent des règles (contraintes, obligations, principes organisateurs, modèles d'action). Pour l'auteur, les systèmes sociaux ne se bornent pas à des actions régies par des intérêts individuels ; ils "reposent toujours sur des règles » qui organisent l'action collective et rendent possibles la cohabitation et la coopération. Opérée pour résoudre des problèmes et les conflits affectant les acteurs, cette régulation fait émerger l'action collective et les acteurs sociaux eux-mêmes. La coexistence des règles est organisée soit par mise en concurrence des légitimités et partage des domaines de pouvoir, soit par la régulation conjointe. Fondée sur la négociation, cette dernière repose sur des règles communes, légitimes pour les parties. Dans le cas des PSE, où existent des divergences et des risques élevés pour les parties, la régulation sociale occupe un rôle central.

\section{Une régulation des PSE inscrite dans un cadre juridique}

L'obligation de réaliser un PSE s'impose aux entreprises de 50 salariés et plus projetant le licenciement pour motif économique de 10 salariés au moins. L'employeur doit (co)financer et mettre en œuvre des dispositifs sociaux et territoriaux atténuant les effets de la restructuration; le PSE regroupe les mesures visant à «éviter les licenciements ou en limiter le nombre ». Il doit intégrer "un plan visant à faciliter le reclassement des salariés dont le licenciement ne pourrait être évité». Encadrée par la DDTEFP compétente, la procédure porte sur les volets économique, social et territorial de l'opération : en interne, l'employeur doit informer et consulter le CE sur le projet de réorganisation (causes, modalités, effets sur l'emploi) et de licenciement (postes supprimés, catégories concernées...) et le PSE projeté. Fondée sur la délégation, la procédure légale repose ainsi sur les représentants des deux principales parties prenantes : la direction et les salariés. Censée favoriser la recherche d'alternatives aux départs et faire évoluer le projet économique, elle se focalise surtout sur la conception, l'amélioration, et le financement du PSE. En externe, quand le projet affecte par son ampleur l'équilibre du bassin d'emploi, l'entreprise doit contribuer financièrement à sa revitalisation. Les acteurs du territoire sont alors impliqués dans l'élaboration et la mise en œuvre du plan de revitalisation.

\section{Des restructurations responsables caractérisées par l'action collective}

Les réflexions actuelles s'orientent vers les restructurations «responsables». Suite aux travaux de la Mission Interministérielle sur les Mutations Economiques, il s'agit d'identifier et partager les bonnes pratiques de gestion des emplois, de conduite des restructurations, et d'accompagnement des personnes. Le dialogue social y est fondamental. Le programme MIRE, le séminaire Restructurations de l'IRES, et l'ouvrage collectif Restructurations d'entreprises, des connaissances pour l'action (Beaujolin-Bellet et Schmidt, 2008), posent l'enjeu de dispositifs équilibrés et collectivement pilotés.

Il en ressort que les jeux d'acteurs et la régulation sociale des restructurations expliquent pour une large part leur acceptabilité pour les parties prenantes, la qualité et la cohérence des PSE, et les résultats atteints, en termes de reclassement ou de solutions "acceptables ». Tout l'enjeu, comme le montrent Bruggeman et al. (2002) puis Aubert et Beaujolin-Bellet (2004), est de passer, dans un contexte de tensions profondes, du mode de la gestion de crise à un pilotage concerté et paritaire des restructurations, dans une logique de projet, pour élaborer de bonnes conditions de reconversion pour les personnes licenciées et les territoires. S'agissant des PSE, le cabinet Syndex a ouvert la voie en montrant que les dispositifs innovants, orientés vers le reclassement, offrant aux bénéficiaires un accompagnement d'une durée suffisante 
(congé de reclassement par exemple), et dépassant le simple catalogue de mesures, peuvent être promus par les RP (Bruggeman et al, 2002). Favoriser l'émergence d'un acteur collectif apparaît ainsi prioritaire. Comme l'explique Beaujolin-Bellet (2003), «il s'agit d'envisager en quoi il est possible de permettre à chaque partie prenante du processus, d'accéder à un véritable statut d'acteur, ni instrumentalisé, ni passif; pour cela, on peut considérer qu'il faut a minima permettre à chaque acteur de disposer de zones d'autonomie en matière de réflexion et d'action, mais aussi, qu'il s'agit de cheminer vers des situations où des combinaisons d'acteurs -l'émergence d'un acteur collectif- sont favorisées ». Outre la coordination des moyens humains et matériels engagés dans le PSE, l'implication des acteurs dépend de la légitimité perçue du projet et de leur pouvoir dans le processus de restructuration. Le partage des diagnostics initiaux, le suivi paritaire des dispositifs et l'évaluation des résultats, y contribuent. Pour reprendre Reynaud (1997), l'enjeu est de passer de la concurrence à la régulation conjointe, propice à l'élaboration de normes (ici, le PSE et son pilotage) légitimes pour les parties. Il s'agit d'instaurer une réelle négociation, mais les règles créées par les échanges entre acteurs et conditions de l'échange sont interdépendantes.

\section{Un contexte peu propice à la régulation paritaire des PSE}

Séduisant en théorie, le pilotage paritaire des PSE se heurte à de nombreux obstacles. Censée, par la consultation des RP, informer les salariés et faire progresser le PSE, la procédure obligatoire est souvent peu productive. Les causes tiennent au contexte social : les inquiétudes suscitées par l'annonce (Dubouloy et Fabre, 2002), les divergences sur la légitimité du projet (Beaujolin-Bellet et al, 2005), le bouclage du dossier avant présentation aux élus, créent un climat délétère, peu propice à la négociation. Elles tiennent aussi au pouvoir des acteurs et à la gouvernance des PSE. Pour Bruggeman et al. (2002), des déséquilibres existent. Ils sont liés à la préparation du projet de restructuration, aux compétences et aux ressources permettant de les acquérir, au pouvoir respectif de la direction et des élus, et à l'appétence au reclassement des parties. Dans les groupes, ils sont amplifiés par la distance entre lieux de décision et lieux d'élaboration des PSE, où les acteurs locaux (direction du site, comité d'établissement) doivent assumer des responsabilités élevées sans la maîtrise des origines de la décision et sans les compétences pour élaborer le PSE (Bruggeman, 2005). Ces déséquilibres tiennent surtout au pouvoir accordé aux RP. Ils disposent d'un droit de regard sur les projets de réorganisation et de licenciement, et sur les mesures d'accompagnement, mais ce pouvoir reste consultatif : le PSE est un engagement unilatéral de l'employeur et le plan de restructuration relève de son pouvoir de décision économique et de sa liberté de gestion.

Ces tensions sont peu productives : elles accouchent de PSE catalogues, conçus par souci de conformité (Mallet et al, 1997), peu adaptés au public concerné, peu évalués (Colin et Rouyer, 1996). Engagée à chaud, la procédure peine à infléchir les choix de réorganisation et à éviter les licenciements (Bruggeman et Paucard, 2003). Il en résulte des déceptions quant aux taux et types de reclassements (Bobbio, 2006). Par ailleurs, la propension de la procédure à impliquer les salariés ne va pas de soi : elle dépend de la représentation collective et des systèmes de délégation établis autour du PSE. Or, des divergences fortes existent souvent entre salariés et RP, et entre RP, sur l'attitude à adopter vis-à-vis de la réorganisation et de l'orientation du PSE. Entre partisans d'indemnités de départ élevées et partisans des aides au reclassement, les oppositions sont fréquentes. L'enjeu est d'élaborer des mesures acceptables par chacun des salariés, volontaire ou désigné, affecté par le projet de licenciement. Leur implication est donc nécessaire à l'élaboration d'un PSE adapté et réaliste.

\subsection{L'apport des tiers à l'élaboration des PSE ? Un focus sur la DDTEFP}

Sous quelles conditions l'acteur collectif peut-il exister? L'émergence d'une régulation collective faisant progresser le PSE dépend en partie de l'élargissement du système d'action à 
des acteurs-tiers. Certains interviennent dans le cadre prévu par la loi, d'autres agissent à la demande ou sous la pression des acteurs déjà engagés. Comme l'expliquent Igalens et Vicens (2008), des parties prenantes habituelles et nouvelles sont impliquées dans les PSE. Parmi ces tierces parties, la DDTEFP reste discrète et son action méconnue.

\section{Un apport de compétences et un effet sur le rapport de force}

Si l'on se focalise sur la procédure obligatoire, les tiers sont des acteurs ou des structures externes, publics ou privés, ayant un rôle d'animation, de financement, de soutien, d'expertise, de contrôle ou d'arbitrage. Les plus importants sont les avocats de la direction, la DDTEFP chargée du dossier, les syndicats, l'expert-comptable du CE, les avocats des RP, les politiques locaux, les juges et les experts en reclassement et revitalisation. Dans les grandes entreprises et les groupes, les dirigeants, parfois les propriétaires, peuvent être sollicités pour donner des garanties ou des justifications. Les RP d'autres sites ou du siège (le CCE en particulier) et les salariés d'autres établissements peuvent apporter des soutiens. Leur intervention dépend de la structure et de la situation de l'entreprise, du financement du PSE, et des jeux d'acteurs noués autour de la procédure.

Sollicitée pour apporter des compétences, des soutiens, ou des moyens, l'intervention des tiers élargit le système d'action concret (Beaujolin-Bellet et Moulin, 2007) et fait évoluer le pilotage du PSE. Elle peut contribuer à équilibrer le rapport de force entre direction et RP, et créer les conditions de la négociation. Les tiers sont des leviers que direction et RP peuvent actionner pour renforcer leurs propres positions (Bruggeman et al, 2002). Les controverses, rendues possibles par leur présence, favorisent le partage des diagnostics sur la situation et l'acceptabilité du PSE (Beaujolin-Bellet et al, 2005). Leur intervention contribue à faire émerger l'acteur collectif (Aubert et Beaujolin-Bellet, 2004), réunissant les acteurs "pertinents », et doté des compétences et moyens propres à améliorer l'intelligence et le contenu du PSE. Au plan symbolique, cette régulation élargie et collective favorise une représentation partagée et positive du PSE (Beaujolin-Bellet et Moulin, 2007). Mais la capacité du système d'action concret à produire des effets vertueux dépend de l'acceptabilité du projet. Elle implique ensuite un minimum de compromis sur l'orientation du PSE. La complexité du système d'action, faisant intervenir des acteurs aux priorités différentes, et fondé sur des délégations multiples (Mazade, 2003) nécessite enfin un pilotage adapté. L'émergence et le maintien de l'acteur collectif dépendent de la volonté des acteurs de coopérer et des structures (cellule de crise, groupe de pilotage...) représentant et coordonnant les parties prenantes. Ces régulations conditionnent l'efficacité du système d'action élargi : 
$\underline{\text { Schéma 1. L'élargissement du système d'action à un ensemble de parties prenantes }}$

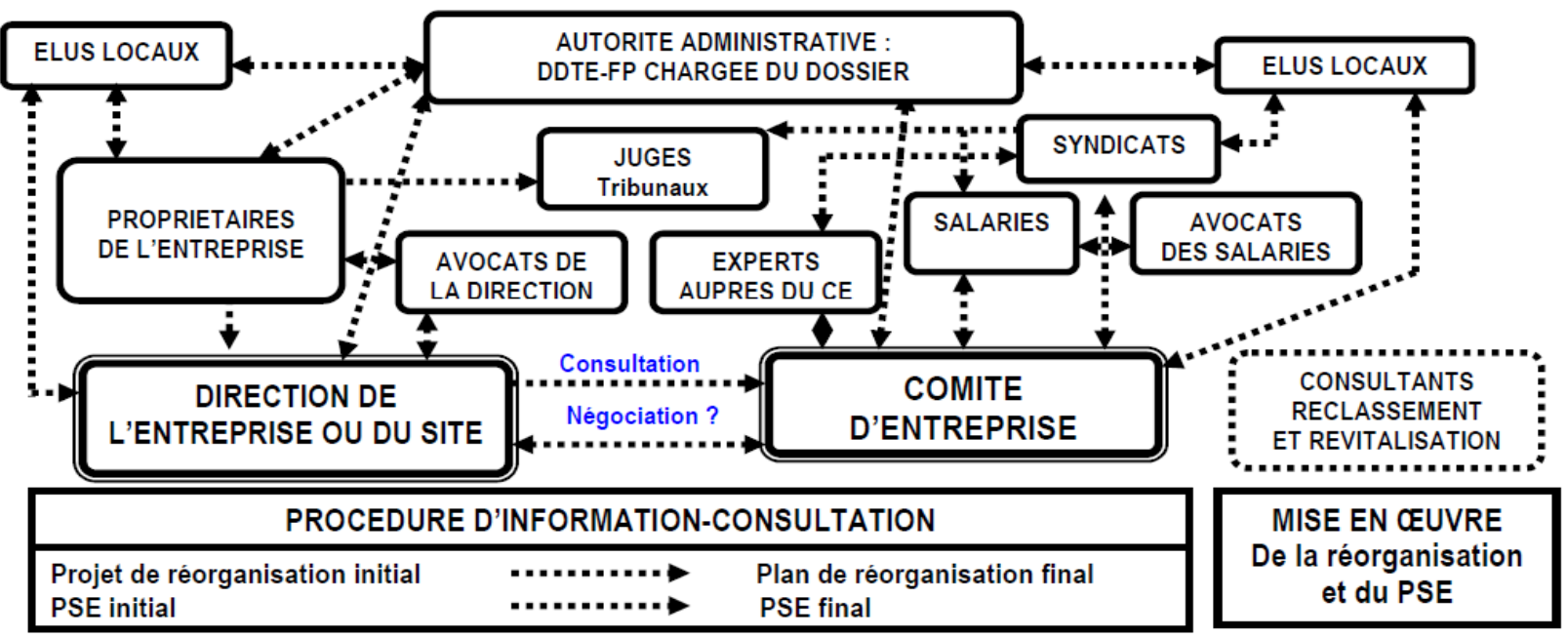

La place accrue des tiers dans les organisations est liée à l'évolution des structures socioproductives (plus dispersées, plus réticulaires), des liens salariés-entreprises (plus fragiles, plus conflictuels) et de l'action de l'Etat sur les territoires (plus décentralisé, plus régulateur). Comme le note Brasseur (2008) "qu'il s'agisse du tiers exclu permanent, interférant ou structurant la relation entre les acteurs ou les organisations, ou du tiers ponctuellement intégré, intervenant pour résoudre une problématique spécifique, le tiers est l'un des leviers de gestion qui bouscule les frontières des organisations et les rôles traditionnels des acteurs ». Leur intervention est justifiée par la gouvernance des contrats "post-fordistes». Orléan (1994) souligne les problèmes de confiance du fait de "l'incomplétude de la logique marchande pure ». Le tiers agit comme garantie pour celui qui doit accorder sa confiance, comme le montre Geindre (2005) à propos du syndicat professionnel dans un réseau stratégique. Dans les relations sociales, l'enjeu est proche, comme l'a illustré le colloque « $L e$ rôle du tiers en gestion »(Cedag, Paris V, 2008) : résolution des conflits, sécurisation des transitions professionnelles, gestion territoriale des $\mathrm{RH}$, relation intérimaire ou gestion du stress, sont autant de situations appelant l'intervention de tiers.

\section{Une problématique et une étude focalisées sur la DDTEFP en charge du dossier}

La DDTEFP a une place majeure dans la procédure obligatoire. Représentant l'Etat, elle assure le contrôle administratif des PSE en jouant sur un double levier juridique et social (Legrand et al, 2003). Son intervention est guidée par trois priorités :

- la conformité du PSE : elle peut dresser un constat de carence "en l'absence de PSE au sens de l'article 1233-61 ». En pratique, la conformité dépend des mesures et moyens engagés en faveur du reclassement.

- la procédure d'information-consultation : il s'agit de contrôler sa régularité. La DDTEFP est informée du projet et reçoit tous les documents produits lors de la procédure (courriers, PV de réunions...). S'il ne peut la suspendre, le Directeur Départemental peut formuler des observations pouvant conforter l'action des RP devant les juges.

- l'amélioration du PSE : durant toute la procédure, la DDTEFP peut faire des propositions pour améliorer le contenu du PSE. Les dirigeants doivent y répondre de façon motivée.

Son rôle va au-delà : à l'interface des parties impliquées, la DDTEFP peut jouer un rôle-clé dans la régulation sociale des PSE, par ses prérogatives et l'expertise apportée. Son action reste méconnue. Les travaux antérieurs privilégient une analyse globale des jeux d'acteurs. 
Parmi les tiers extérieurs, l'attention est surtout portée sur l'expert-comptable du CE, ses effets sur le rapport de force direction/RP et la construction de l'acceptabilité du PSE (Paucard et al 2006 ; Beaujolin-Bellet et Moulin, 2007). La régularité du PSE étant un levier du rapport de force, le rôle de la DDTEFP est abordé dans ce cadre (Bruggeman et al., 2002).

Sur la base de ces constats, notre problématique est la suivante : dans quelle mesure et à quelles conditions l'intervention de la DDTEFP peut-elle contribuer à la régulation sociale et au contenu des PSE?

Pour y répondre, nous nous appuyons sur l'étude de trois PSE, menée dans une recherche sur l'accompagnement social des restructurations pour la DRTEFP du Languedoc-Roussillon. Il s'agissait d'analyser et évaluer les dispositifs engagés "à chaud», via les PSE, et des démarches d'anticipation engagées « à froid». La partie de l'étude restituée ici se focalise sur l'élaboration du PSE de l'annonce officielle à la clôture de la procédure. La mise en œuvre n'est pas abordée mais nous pensons qu'elle dépend fortement de la phase amont.

Une analyse multi-acteurs a été menée (58 entretiens) : responsables DDTEFP (directeurs départementaux (DD), membres des services « restructurations » chargés du suivi des PSE) et DRTEFP (service "mutations économiques », chargés d'études), dirigeants des entreprises concernées, membres du CE et/ou délégués syndicaux, experts-comptables du CE, consultants chargés du reclassement et de la revitalisation, responsables ANPE membres des commissions de suivi, et, si possible, le Préfet ou son représentant et le Maire de la commune. C'est dans ce cadre que l'action de la DDTEFP a été observée.

Les entretiens ont fait l'objet d'une analyse qualitative individuelle et collective pour faire émerger les éléments-clés de la régulation sociale et de l'évolution chronologique des PSE. Par recoupements de données, l'analyse multi-acteurs fait émerger les faits saillants et limite les biais liés de représentations. Les entretiens sont complétés par l'analyse des documents produits (dossier économique, projet de PSE et PSE final, courriers direction-DDTE, PV de réunion direction-CE) qui permettent d'objectiver et renforcer l'analyse des cas. Les analyses ont été discutées avec les trois DD et les adjoints des services «entreprises » ayant suivi les dossiers.

\section{Les résultats de l'étude : l'action des DDTEFP, décisive mais contingente}

Dans un premier temps, nous présentons les trois cas et examinons l'évolution des jeux d'acteurs et des PSE élaborés (2.1). Nous analysons ensuite la contribution des DDTEFP à la régulation sociale des PSE et les conditions propices à l'efficacité de leur action (2.2).

\subsection{L'analyse chronologique des cas : l'évolution du système d'action concret et du contenu des PSE}

Les cas ont été choisis selon leur actualité (PSE récents, négociations bouclées, reclassements achevés ou en cours), leur localisation et l'accès aux acteurs pertinents. Ils se situent dans trois départements et sont pris en charge par des DDTEFP distinctes. Ils sont différents (de liquidation pour Trans, sauvegarde pour Déco et Véti) mais sont présentés pour leurs apports à l'analyse de la régulation des PSE. Le contexte initial, économique, social et territorial, est fondamental pour comprendre ce qui suit. Les cas ont été anonymés (noms et lieux).

\section{Présentation des trois cas et des contextes de départ}

VETI fabrique des produits d'habillement. Elle compte 600 salariés avant le PSE étudié, dont 500 à Lautun, ville rurale où se situe la production. L'actionnaire principal est reconnu pour ses «valeurs» : "c'est un opérateur industriel, pas un fonds de pension » dit un dirigeant. Les difficultés économiques s'accumulent : la consommation baisse, la concurrence s'accroît, 
le rapport aux grandes surfaces est défavorable. La réorganisation annoncée affecte 158 postes, surtout en production. Il s'agit pour l'essentiel de femmes d'une ancienneté élevée, habituées au travail posté. Il est prévu des départs volontaires dans les groupes d'emplois en sureffectif. Véti affiche une culture sociale forte : selon le DRH, «la culture paternaliste d'origine s'est estompée mais les liens entre direction et salariés en sont encore teintés ». La politique RH est développée et les conditions d'emploi avantageuses, les syndicats sont présents et expérimentés. Le CCE recourt à un expert-comptable qui connaît bien l'entreprise. Le contexte territorial est défavorable: le bassin d'emploi rural est fragile, Véti reste la dernière grande entreprise.

DECO est un groupe industriel international spécialisé dans les produits d'aménagement et de décoration. Le siège est situé en Amérique du Nord. Les principaux actionnaires sont des fonds de pension, d'où la distance entre lieu de décision et lieux de production. Déco connaît des difficultés à partir des années 1990. Les problèmes sont tels que le groupe est mis en redressement dans le cadre du Chapter 11 américain. L'analyse des syndicats est explicite: "cela fait cinq ans que nous sommes maintenus sous perfusion. Nous n'avons pas su nous diversifier. Nos dirigeants ont choisi de privilégier la marque. Nos produits sont chers par rapport à la concurrence ». La direction Europe décide de fermer St-Jean. La syndicalisation est forte (2/3 de syndiqués en 2000) mais s'est émoussée (30\% aujourd'hui) et transformée (le syndicat majoritaire pendant 30 ans a perdu sa place). Le PSE affecte 150 emplois. Le contexte territorial est jugé très défavorable. Des fermetures ont causé la quasi-disparition de la filière industrielle, une baisse démographique et la disparition de services publics.

TRANS est un groupe régional spécialisé dans le transport aérien. La spécificité du métier, le contexte économique, psychologique, et politique, et le nombre de licenciements, en font un cas hors normes. Créée en 1972, son historique est mouvementé avec de nombreux changements dans la structure de propriété. La compagnie est à l'agonie. Plusieurs repreneurs se manifestent mais le plan de reprise du dernier est finalement annulé. La décision de liquidation du groupe provoque un choc violent. Le PSE de fermeture, piloté par le mandataire-liquidateur (et non par les dirigeants) concerne 600 personnes. La syndicalisation est forte. La culture de l'entreprise est un élément-clé : si le groupe est décrit comme "un archipel de sous-cultures », les salariés sont animés par une passion commune pour le métier et l'entreprise. Le contexte territorial est favorable: bassin urbain dense, grande ville dynamique. Le problème tient à la faiblesse des débouchés locaux liée à la forte spécialisation d'une partie du personnel.

Dans les trois départements, le suivi administratif est identique : le contrôle de la procédure, de l'annonce du projet à la clôture du PSE, est assuré par les DD : "les grands licenciements et les PSE sont forcément prioritaires » explique l'un d'eux. Au sein des DDTEFP, deux organes sont à distinguer : "le service restructuration donne un avis qualitatif sur les mesures prévues et suivent, par délégation, la mise en ouvre des PSE, l'Inspection intervient en amont de la procédure et lors des licenciements pour veiller au respect du droit du travail. Ils relèvent de deux métiers distincts : l'emploi et le travail » note une responsable.

\section{L'évolution des jeux d'acteurs et du contenu des PSE}

Nos observations confirment celles de Bruggeman et al. (2002) quant aux déséquilibres initiaux. Les difficultés sont avérées mais les projets de réorganisation, préparés dans le secret, provoquent des chocs violents. Les argumentaires des directions sont stéréotypés se limitant aux causes légales (dégradation du résultat, manque de débouchés, sauvegarde de la compétitivité). Les véritables buts et les alternatives possibles sont peu lisibles, surtout lorsque les lieux de décision sont éloignés (Déco). Il en découle une difficulté à réaliser un diagnostic serein et, pour les salariés, à accepter la réorganisation; chez Véti, le plan de 
licenciement est jugé excessif, certains élus estimant possible le maintien de l'activité ; chez Déco, il est reproché au groupe d'avoir condamné St-Jean pour favoriser d'autres sites ; chez Trans, malgré la situation, aucun salarié ne croit à la liquidation. Pour un dirigeant de Véti, anticiper suppose une volonté commune de la direction et des élus: "quand une restructuration est prévue, il faut informer, donner des signes. Par exemple, faire une semaine à zéro à un moment où d'habitude les ateliers fonctionnent à plein régime mais si les $R P$ refusent de signer un accord pour anticiper, il est impossible d'annoncer le projet à l'avance puisque l'annonce déclenche la procédure obligatoire ». Les procédures débutent dans un climat de tensions, mais connaissent des évolutions différentes :

VETI : l'annonce du projet crée de fortes tensions (menaces, pneus crevés, manifestations...). Un rapport de force s'installe entre direction et RP, ces derniers jouant de tous les leviers. Pour le DRH, "le PSE est un moyen de réorganisation nécessaire mais dangereux, il provoque de fortes ruptures sociales. Il y a beaucoup de violence, les personnes ont peur. Dans les premiers moments, il y a beaucoup d'irrationnel ». Les premières réunions direction/CE/CCE sont très tendues. Mais les entretiens menés et les PV de réunions montrent que les élus, aidés par l'expert-comptable, admettent peu à peu le caractère inévitable des licenciements. Un consensus tacite se dégage sur les efforts à fournir pour faire progresser le PSE. Ces échanges «musclés mais responsables » ont permis de dépasser le rapport de force : «chacun a joué son rôle, des relations constructives ont été nouées tout au long de la procédure, malgré les tensions » estime un dirigeant. La prise de contact très tôt avec la DDTEFP pour l'informer du projet, "valider» la procédure prévue et connaître les soutiens publics possibles, est un élément à souligner. Un RP estime que le DD s'est imposé comme " une autorité incontournable encadrant la procédure de façon rigoureuse et omniprésente ». Un autre souligne "la qualité de la collaboration qui a pu s'établir entre le DD, les RP et les experts $d u C C E$ ». Le DD avoue avoir usé de son pouvoir d'influence pour "faire comprendre aux dirigeants qu'en deçà de certaines mesures le constat de carence était possible ». L'obtention du co-financement par l'Etat d'un congé de conversion, dispositif exceptionnel, est un moment-clé dans la procédure, un dispositif central dans le PSE. Rendu possible par la démarche du DD auprès des autorités, justifié par la fragilité des salariés et du territoire, il débloque la procédure en offrant un compromis sur le contenu du PSE.

DECO : le projet de fermeture provoque un état de crise. Le personnel se mobilise, des actions sont organisées: AG, manifestations, journées ville morte, démarches auprès des responsables politiques, grève du zèle... La médiatisation est forte. Sous la pression des RP et de la DDTEFP, le PSE initial, jugé insuffisant quant aux dispositifs de reclassement prévus, devient caduc et la procédure est reprise à zéro. Les réunions entre élus et direction sont tendues, d'autant que le rapport de l'expert-comptable met en cause le bien-fondé de la fermeture du site. Mais les efforts convergent peu à peu pour soutenir le dispositif de reclassement : «de toutes façons, la fermeture était inéluctable, la décision du groupe était ferme. Il était important de calmer les esprits, de faire notre possible pour que le reclassement se passe au mieux » résume un élu. Très présent dès le début, le DD a fortement encadré la procédure, suivi les réunions direction/RP et fait des propositions pour améliorer le PSE, comme en attestent les courriers échangés avec la direction. Dans une logique donnantdonnant, il pousse la direction à renforcer le dispositif de reclassement (allongement de la durée du congé de reclassement) et fait des concessions sur le nombre de préretraites ASFNE, le financement de la cellule de reclassement au-delà de la durée du congé, et l'aide à la création d'entreprise. L'obtention de moyens complémentaires auprès des collectivités (enveloppe-formation débloquée par le conseil régional et postes spécifiques ouverts par le conseil général) aboutit à un compromis sur le contenu du PSE. 
TRANS : la période précédant la liquidation est peu conflictualisée. Le flou de la situation économique et financière, l'espoir jusqu'au bout d'une reprise et la crainte de dissuader les repreneurs, ont limité et dilué l'action des salariés et de leurs représentants. Le DD souligne « un manque de clairvoyance d'une partie du personnel vis-à-vis de la situation réelle et des risques pour leur emploi ». La liquidation provoque un choc violent. Les salariés sont très touchés affectivement (inquiétude, tristesse, abattement, dépressions, suicides...) et socialement (crainte de "ne plus pouvoir faire face ", "ne pas retrouver quelque chose de décent » selon un élu). La DDTEFP est saisie tardivement, une fois la liquidation actée. La procédure est menée dans l'urgence et la souffrance. Les RP sont désorganisés et divergent quant à la conduite à adopter. En l'absence de l'employeur défaillant, la DDTEFP est le pivot du PSE et des mesures qui l'accompagnent. Des moyens exceptionnels sont obtenus auprès des collectivités et des pouvoirs publics (formations spécifiques, maintien des compétences, facilités de transport...). Mais l'intervention de la DDTEFP, ex post, a peu d'impact sur la procédure elle-même et sur la mise en place des dispositifs du PSE.

L'élargissement du système d'action permet dans deux des trois cas (Véti et Déco) l'évolution du pilotage et du contenu des PSE. Les avancées obtenues concernent surtout le volet social du projet et le dispositif de reclassement externe, qui reste la priorité des DDTEFP. Les autres mesures d'accompagnement (mutations internes, mesures d'âge, indemnités financières, enveloppes-formation) progressent aussi, grâce aux efforts consentis par la direction ou aux soutiens obtenus auprès des collectivités, et renforcent la consistance des PSE. En revanche, le projet de réorganisation échappe au pouvoir des RP, même si dans un cas (Véti) le nombre de licenciements est revu à la baisse.

\section{Tableau 1. Les avancées obtenues au cours de la procédure}

\begin{tabular}{|c|c|}
\hline VETI & $\begin{array}{l}\text { - Limitation des départs grâce à des mutations internes ( } 45 \text { postes sur les } 158 \text { supprimés) } \\
\text { - Congé de conversion (exceptionnel) après accord DDTEFP et direction (co-financement 50/50) } \\
\text { - Allongement de la durée de la cellule de reclassement de } 9 \text { à } 12 \text { mois } \\
\text { - Convention de cellule de reclassement adossée au congé de conversion (co-financement } 50 / 50 \text { ) } \\
\text { - Evolution du dispositif de passage à temps partiel ( } 25 \% \text { au lieu de } 33 \%) \\
\text { - Augmentation de la prime de licenciement }\end{array}$ \\
\hline DECO & $\begin{array}{l}\text { - Augmentation du nombre de préretraites accordées par la DDTEFP } \\
\text { - Limitation des départs par des propositions de mutation (12 postes en France et } 28 \text { à l'étranger) } \\
\text { - Allongement de la durée du congé de reclassement de } 6 \text { à } 9 \text { mois } \\
\text { - Augmentation de la prime d'aide à la création d'entreprise et de l'indemnité de licenciement } \\
\text { - Indemnité pour préjudice moral versée à l'ensemble des salariés } \\
\text { - Prise en charge par la Région (co-financement FSE) des dépenses de formation } \\
\text { - Ouverture de postes par le Conseil Général (concours « réservé ») }\end{array}$ \\
\hline TRANS & $\begin{array}{l}\text { - Mesures financées par l'Etat : cellule de reclassement, allocations temporaires dégressives, aide } \\
\text { à la création ou à la reprise d'entreprise } \\
\text { - Moyens exceptionnels obtenus grâce au soutien de l'Etat et des collectivités : } \\
\text { - Maintien des compétences (co-financement ANPE/Assedic de bilans de compétences, et co- } \\
\text { financement DG Transports/ branche professionnelle de prorogations de qualifications) } \\
\text { - Fonds régional de soutien au reclassement (co-financement FSE sur une partie) } \\
\text { - Prorogation des qualifications techniques (co-financement par la DDTE-FP et l'Assedic) } \\
\text { - Facilités de transport accordées aux salariés en recherche d'emploi }\end{array}$ \\
\hline
\end{tabular}

\subsection{Discussion : quelle contribution des DDTEFP à la régulation des PSE ?}

En tant qu'autorité administrative, la DDTEFP doit veiller au bon déroulement de la procédure et à la conformité du PSE. Le DD en charge de Trans explique : "nous avons deux niveaux de préoccupations : limiter au maximum le nombre de licenciements et favoriser le 
reclassement interne et externe des salariés licenciés. Toute notre intervention est centrée autour de ces objectifs. Il s'ensuit une négociation avec la direction et les RP que nous essayons de favoriser et d'accompagner au mieux ». La DDTEFP apparait ainsi comme un acteur-tiers particulier, en même temps extérieur au rapport de force et interface entre les parties prenantes impliquées. Son rôle oscille entre contrôle administratif externe a posteriori et intervention continue «au cœur» de la procédure. Son action peut contribuer à rééquilibrer les rapports de force, à finaliser et à renforcer le PSE. Mais sa contribution est elle-même contingente des régulations sociales qui entourent le PSE.

\section{Une contribution entre autorité, médiation et expertise}

Le rapport de force présent au début de la procédure est à double tranchant. S'il renforce les positions des protagonistes, il produit des tensions et des ruptures parfois irréversibles. Le bon déroulement de la procédure dépend en partie de sa régulation. La DDTEFP peut y jouer un rôle-clé, en premier lieu, en tant qu'arbitre extérieur, par l'application des règles légales. Le contrôle administratif du bon déroulement de la procédure (respect des délais, du nombre de réunions, des ordres du jour...) et de la conformité du PSE, sont les deux premiers leviers. Chez Véti et Déco, la «surveillance » des DD est précoce et continue : de l'avis de tous, la conduite et le contenu du PSE sont "fortement cadrés». Chez Trans, dans un contexte de liquidation, l'action du DD se focalise sur le PSE. La procédure est menée en quelques semaines, dans les délais minima, son rôle en amont est limité.

Ce rôle d'arbitre, «de gendarme », n'est que la partie visible de son action. A l'interface des parties prenantes, la DDTEFP joue aussi un rôle de médiateur: "en tant qu'autorité administrative compétente, nous avons des contacts avec les dirigeants, les salariés, les syndicats. Nous sommes sollicités pour informer, arbitrer, trouver des moyens. Beaucoup de coups de téléphone, beaucoup d'informel, beaucoup d'ajustements » explique le DD en charge de Déco. Pour reprendre Orléan (1994), elle peut apporter, par son autorité et sa position, une "garantie » nécessaire à la construction de compromis entre protagonistes. Cette régulation suppose que la DDTEFP ne se contente pas d'imposer des règles légales, mais qu'elle permette aux parties prenantes d'élaborer leurs propres règles, adaptées à leurs besoins et légitimes pour elles, à l'intérieur de ce cadre. Dans deux des trois cas, elle est à l'origine des compromis sur les dispositifs qui forment le cœur des PSE (congé de conversion pour Véti, congé de reclassement pour Déco). Ces compromis sont liés en partie au financement du PSE : en conditionnant les aides publiques aux efforts de l'employeur, le DD dispose d'un levier pour le faire progresser. L'expertise apportée par les DDTEFP doit enfin aussi être soulignée : même si chacune a ses habitudes, une «jurisprudence » oriente leurs actions quant au contenu, à la cohérence, et au pilotage des PSE et quant aux multiples conventions qui lient l'entreprise, l'Etat, les prestataires et les RP.

\section{Une contribution contingente, tributaire du contexte et des jeux d'acteurs}

La contribution de la DDTEFP à la régulation et au contenu des PSE est variable. Elle dépend d'abord du moment et du degré de son implication. Les cas de Véti et Déco, où elle intervient très tôt et de façon continue, et celui de Trans, où elle intervient ex-post et de façon plus ponctuelle, sont très différents. Dans les premiers cas, elle a contribué à l'équilibrage du rapport de force et du système de pilotage, beaucoup moins dans le second où son action se focalise sur le contenu du PSE. Le moment de son intervention dépend de l'instant où elle est avertie mais aussi de l'importance objective du dossier: "quand nous avons eu à gérer ce PSE, compte tenu de son poids, il a été prioritaire sur les autres » explique le DD qui suit Trans. Des facteurs subjectifs jouent aussi : "C'est injuste, mais l'implication des pouvoirs publics dépend des remous sociaux et médiatiques » explique un autre responsable. 
Elle dépend ensuite de la personnalité du DD. Un dirigeant de Déco le souligne avec humour : «au début, nous avions beaucoup de craintes à l'égard des attentes du représentant DDTEFP. Il fallait toujours argumenter, justifier, chiffrer, améliorer nos indicateurs ». Le DRH de Véti affirme que «le DD a été proactif, omniprésent, c'est un homme déterminé, pugnace ». Le DD en charge de Déco conclut «si l'implication de l'administration va de soi (elle est prévue et cadrée par la loi), son intensité dépend beaucoup des personnes, c'est comme partout, ce sont les personnes qui font que ça marche ou pas ».

Elle dépend aussi des marges de manœuvre financières de l'entreprise et de la DDTEFP. Quand l'entreprise est «en mesure de payer », la DDTEFP peut peser sur l'implication de l'employeur en conditionnant l'acceptation du projet et la participation financière de l'Etat à la qualité du dispositif proposé. Mais ce n'est pas toujours possible. Le DD en charge de Déco explique par exemple que "quand un PSE arrive en fin d'année budgétaire, il ne reste souvent pas grand-chose. C'est inégalitaire, c'est sûr, mais c'est une réalité ».

Elle dépend enfin des régulations sociales entourant le PSE. L'étude confirme l'importance de l'acteur collectif soulignée par Beaujolin-Bellet (2003). Il est constitué d'acteurs individuels qui élaborent ensemble des règles et des solutions face à un problème qui les affecte (Reynaud, 1997). La régulation est alors conjointe et paritaire, la rationalité sociale et non légale. Le DD en charge de Trans note qu' "au-delà du socle commun de mise en ouvre, tout se joue dans la conviction et l'implication des individus, l'administration ne peut pas tout toute seule, la mobilisation des salariés est un facteur essentiel, les évolutions du contenu du PSE résultent de la combinaison des différents acteurs ». Chez Véti et Déco, différents facteurs y ont contribué. Au départ, la responsabilité des acteurs et la volonté de progresser pour trouver des compromis satisfaisants sont mises en avant. La condition fondamentale est l'acceptabilité de la restructuration. Elle repose sur la légitimité du projet économique et les moyens pour en atténuer les impacts sociaux, mais aussi sur la capacité donnée aux parties prenantes de défendre leurs intérêts et influer sur le projet. La dimension procédurale de la justice organisationnelle joue ici un rôle central. Mais «tout ne se joue pas pendant le PSE » note un dirigeant; la confiance nécessaire au dialogue dépend de la culture et des relations sociales préalables. Ensuite, lors de l'élaboration du PSE, l'instauration de systèmes de pilotage paritaires est déterminante. Il s'agit surtout d'associer les RP au choix et à la conception des dispositifs d'accompagnement. Leur soutien est d'autant plus fort qu'ils y sont associés très en amont. Le rôle des élus est essentiel pour informer les salariés, les convaincre de l'intérêt des mesures de reclassement. Lorsque les élus ont adhéré, leur action auprès des salariés a été décisive : explications sur les avancées, rencontres individuelles... Dans le cas de Trans, l'absence perçue de légitimité du projet et l'état psychologique des salariés ont pesé jusqu'au bout. Malgré les moyens exceptionnels engagés, le taux de reclassement est inférieur à $30 \%$, CDD compris...

\section{Conclusion}

A l'interface des différentes parties prenantes, la DDTEFP peut jouer un rôle de médiation, d'arbitrage, et de garantie, propres à canaliser et équilibrer les rapports de force. Par son expertise et les financements obtenus, elle peut aider à améliorer le PSE. Son action participe de l'émergence de l'acteur collectif mais elle est tributaire des jeux d'acteurs qui entourent le PSE. A cheval entre conflit et coopération, ces régulations sont complexes et fragiles. L'instauration d'une dynamique de négociation dépend des relations préalables direction-élus et de leur volonté de coopérer, mais elle ne semble pas pouvoir faire l'économie d'un rapport de force. Les moyens obtenus dépendent de la capacité des protagonistes à médiatiser le PSE et impliquer des tiers extérieurs. Aux inégalités de droit s'ajoutent ainsi des inégalités de fait ; 
il est regrettable de constater que la conflictualisation de la situation soit une des conditions de leur mobilisation (y compris des pouvoirs publics) et des moyens consacrés au PSE.

Nos résultats sont à relativiser. Le nombre de cas observés n'autorise pas la généralisation à l'ensemble des PSE et leur sélection comporte certains biais. L'étude peut toutefois éclairer la réflexion sur l'accompagnement des restructurations. La complexité et l'urgence des situations économiques et sociales, individuelles et collectives, nécessitent un rapprochement et une coordination des expertises. Or, la spécialisation des acteurs publics d'un côté, et les conflits politiques de territoires de l'autre, créent un émiettement peu propice à l'action collective. De ce point de vue, la mise en place de structures permanentes de dialogue et de veille sur les restructurations et d'accompagnement des mobilités est à préconiser.

En termes de régulation sociale, l'étude révèle un paradoxe : quand elle existe, la négociation se borne au volet social, c'est-à-dire au PSE proprement dit, le projet économique relevant des prérogatives de la direction. Autrement dit, si régulation conjointe il y a, elle s'organise dans un partitionnement des pouvoirs. C'est une des raisons pour lesquelles certaines instances représentatives refusent de s'engager dans la négociation sur les PSE. L'obligation de négociation triennale sur la GPEC, réservée aux grandes entreprises, n'y répond qu'en partie.

\section{Bibliographie}

AUBERT J.P., BEAUJOLIN-BELLET R. (2004), Les acteurs de l'entreprise face aux restructurations : une délicate mutation, Travail et Emploi, $\mathrm{n}^{\circ} 100$, oct., p.99-112.

BEAUJOLIN-BELLET R., SCHMIDT G. (coord.) (2008), Restructurations d'entreprises, des connaissances pour l'action, Vuibert.

BEAUJOLIN-BELLET R., MOULIN Y. (2007), Des dispositifs de reclassement : une évaluation par les acteurs contingente aux modalités de leur construction, $\mathrm{XI}^{\mathrm{e}} \mathrm{J}^{\mathrm{e} e s}$ Internationales de Sociologie du Travail, juin.

BEAUJOLIN-BELLET R., BRUGGEMAN F., PAUCARD D. (2005), Décisions de restructuration et jeux d'acteurs : la construction de l'acceptabilité sociale des licenciements accompagnés de plans sociaux, Management et Avenir, $\mathrm{n}^{\circ} 9, \mathrm{p} .65-81$.

BEAUJOLIN-BELLET R., (2003), Le pilotage des restructurations : 1'innovation est dans les processus, 14è Congrès de l'AGRH.

BOBBIO M. (2006), Les PSE : accompagner les salariés licenciés sans garantie de retour vers l'emploi stable, DARES, PIPS, 28.2.

BRASSEUR M. (2008), Le rôle du tiers en gestion, CEDAG, Paris V, juin.

BRUGGEMAN F. (2005), Plans sociaux : l'impossible accompagnement social des licenciements économiques?, IRES, n 47 , p.215-231.

BRUGGEMAN F., PAUCARD D. (2003), Un bilan des pratiques françaises et du dispositif d'accompagnement des restructurations, Séminaire sur les restructurations responsables, Dublin.

BRUGGEMAN F., LAPOTRE M., PAUCARD D., THOBOIS M. (2002), Plans sociaux et reclassement : quand l'innovation est promue par les représentants des salariés, DARES.

COLIN T., ROUYER R. (1996), La loi sur les plans sociaux face aux logiques gestionnaires, Travail et Emploi, n 69 , p.5-22.

DUBOULOY M., FABRE C. (2002), Les restructurations d'entreprises : de la rationalité économique à la souffrance des hommes, Gérer et Comprendre, mars, p.43-55.

GAZIER B. et SCHMID G. (2002), The new dynamics of full employment. Social integration through labour markets in the european union, E.Elgar.

GEINDRE S. (2005), Le rôle de l'acteur-tiers dans la construction d'un réseau stratégique, RFG, $\mathrm{n}^{\circ} 154$, p.75-91. 
IGALENS J. et VICENS C. (2008), Contribution de la théorie des parties prenantes à une approche théorique et normative des restructurations, in BEAUJOLIN-BELLET et SCHMIDT (coord.), Restructurations d'entreprises, des recherches pour l'action, Vuibert, p.103-120.

LEGRAND B., DE TAILLAC L., TERRACOL A. (2003), L'intervention de l'Etat dans l'accompagnement des plans sociaux, Regards sur l'Actualité, $\mathrm{n}^{\circ} 290$.

MALLET L., REYNES B., TESSIER F., VICENS C. (1997), A quoi servent les plans sociaux ? Travail et Emploi, ${ }^{\circ} 72$, mars, p.79-99.

MAZADE O. (2003), Reconversion des salariés et plans sociaux, L'Harmattan.

ORLEAN A. (1994), Sur le rôle respectif de la confiance et de l'intérêt dans la construction de l'ordre marchand, revue du MAUSS, 2̇̀ sem., p.17-36.

PAUCARD D., BRUGGEMAN F., VERKINDT P. Y. (2006), Restructurations et expertises, Droit Social, n³, mars, p.334-340.

REYNAUD J.D. (1997), Les règles du jeu. L'action collective et la régulation sociale, A.Colin. 